\title{
DUALITY THEOREMS AND THEOREMS OF THE ALTERNATIVE
}

\author{
L. MCLINDEN ${ }^{1}$
}

ABSTRACT. It is shown, in a completely general setting, that a theorem of the alternative is logically equivalent to a duality theorem linking two constrained optimization problems.

A standard technique for proving a duality theorem linking two constrained optimization problems is to apply an appropriate theorem of the alternative, sometimes called a transposition theorem (see, e.g., Mangasarian [2], Rockafellar [4], Stoer and $\mathbb{V}$ itzgall [6]). In the context of linear problems in finite dimensions it has been observed also by some (e.g., Balinski and Tucker [1]) that, conversely, from such a duality result a theorem of the alternative follows. In this note we extract the essence of the logical arguments involved in such derivations and thus exhibit the extreme generality of these techniques of proof. In particular, we show the simple, yet basic, logical principle that a duality theorem is actually equivalent to a theorem of the alternative. No linear space structure is needed.

Let $X$ and $Y$ be arbitrary nonempty sets, and let $f$ and $g$ be arbitrary extended-real-valued functions defined on $X$ and $Y$, respectively. For each $a \in(-\infty,+\infty]$ consider the statements

$$
\begin{aligned}
& \left(\mathrm{I}_{a}\right) \quad \exists x \in X \text { such that } f(x)<a, \\
& \left(\mathrm{II}_{a}\right) \quad \exists y \in Y \text { such that } g(y) \geq a .
\end{aligned}
$$

The following statement is an abstract theorem of the alternative involving the pairs $(f, X)$ and $(g, Y)$ :

$$
\left.\forall a \in(-\infty,+\infty] \text {, exactly one of }\left(\mathrm{I}_{\alpha}\right), \text { (II }{ }_{a}\right) \text { holds. }
$$

We do not assert anything here as to the validity of $(\mathbb{P})$, but merely introduce it as a logical statement. Consider now the two abstract optimization problems

$$
\inf _{x \in X} f(x) \text { and } \sup _{y \in Y} g(y)
$$

Received by the editors September 13, 1974.

AMS (MOS) subject classifications (1970). Primary 90C99; Secondary 15 A39.

Key words and phrases. Transposition theorems, dual extremum problems.

${ }^{1}$ Sponsored in part by Contract DA-31-124-ARO-D-462 at the Mathematics Research Center, University of Wisconsin-Madison. 
The fact that $f$ and $g$ are allowed infinite values permits constrained problems to be viewed in this format. For these problems', the following logical statement is an abstract duality theorem:

$$
\inf _{x \in X} f(x)=\max _{y \in Y} g(y) .
$$

We understand this to mean that $\inf _{X} f=\sup _{Y} g$ and $g(y)=\sup _{Y} g$ for some $y \in Y$.

Of course, neither $(\mathscr{P})$ nor $(\mathcal{T})$ has any real content until $(f, X)$ and $(g, Y)$ are assigned specific interpretations, or structure, and also hypotheses are given under which $(\mathfrak{Q})$ or $(\mathfrak{T})$ is indeed true. For instance, in abstract terms, the theory of dual optimization problems involves first representing $f$ (say) in terms of some other function $L$ of the form $L: X \times Y$ $\rightarrow[-\infty,+\infty]$ by means of the relation

$$
f(x)=\sup _{y \in Y} L(x, y), \quad \forall x \in X,
$$

and then taking $g$ to be

$$
g(y)=\inf _{x \in X} L(x, y), \quad \forall y \in Y .
$$

In this case $\inf _{X} f$, the primal problem, corresponds to half of the minimax problem determined by $L$, while the dual problern $\sup _{Y} g$ corresponds to the other half. The duality theorem $(\mathcal{T})$ can then be rewritten equivalently as an abstract minimax theorem for $L$ :

$$
\inf _{x \in X} \sup _{y \in Y} L(x, y)=\max _{y \in Y} \inf _{x \in X} L(x, y) .
$$

The basic logical principle we wish to record is the following.

Proposition. ( $(\mathfrak{Q})$ holds if and only if $(\mathfrak{T})$ holds.

The "if" half of the Proposition, when combined with the framework just mentioned for dual optimization problems (see, e.g., Rockafellar [4], [5]), furnishes an extremely general recipe for theorems of the alternative. Indeed, starting out with some appropriate set of hypotheses on $f$ or $g$ (or $L$ ) which validate statement $(\mathcal{P})$ (or $(\mathfrak{T})$ ), one then concludes that the corresponding statement $(\mathfrak{Q})$ is valid under exactly the same set of hypotheses. A forthcoming paper [3] will show how this technique can be used to derive new transposition theorems virtually on demand as well as extend a number of the classical ones to broader, infinite-dimensional and nonpolyhedral settings.

The proof of the Proposition is immediate from two lemmas, which may be of interest in their own right as they provide a more detailed comparison 
of $(\mathcal{C})$ and $(\mathcal{T})$. In what follows the symbol $\sim$ denotes negation.

Lemma 1. The following two statements are equivalent:

$$
\forall \alpha \in(-\infty,+\infty],\left(\mathrm{II}_{\alpha}\right) \text { implies } \sim\left(\mathrm{I}_{\alpha}\right) \text {; }
$$

$$
\inf _{x \in X} f(x) \geq \sup _{y \in Y} g(y) .
$$

Lemma 2. The following two statements are equivalent:

$$
\forall a \in(-\infty,+\infty], \sim\left(\mathrm{I}_{\alpha}\right) \text { implies }\left(\mathrm{II}_{\alpha}\right) \text {; }
$$

$$
\exists y \in Y \text { such that } g(y) \geq \inf _{x \in X} f(x) .
$$

Observe that $\left(d_{1}\right)$ is the so-called weak duality theorem for the two optimization problems, while $\left(\mathrm{a}_{2}\right)$ corresponds (albeit in a highly abstract way) to the nontrivial half of the theorem of the alternative.

It is clear that $(\mathbb{P})$ is equivalent to $\left(a_{1}\right)$ and $\left(a_{2}\right)$, and also that $(T)$ is equivalent to $\left(d_{1}\right)$ and $\left(d_{2}\right)$. Thus, the Proposition will be proved once the two lemmas are.

Proof of Lemma 1. To see that $\left(a_{1}\right)$ implies $\left(d_{1}\right)$ : Let $y \in Y$ and put $a=g(y)$. If $\alpha=-\infty$, $\inf _{X} f \geq-\infty$ trivially. If $\alpha \in(-\infty,+\infty],\left(a_{1}\right)$ implies $\inf _{X} f \geq a$. Since $y \in Y$ was arbitrary, $\left(\mathrm{d}_{1}\right)$ follows. To see that $\left(\mathrm{d}_{1}\right) \mathrm{im}$ plies $\left(a_{1}\right)$ : Let $a \in(-\infty,+\infty]$ be such that (II $\left.{ }_{a}\right)$ holds. Then $\left(d_{1}\right)$ implies $\inf _{X} f \geq \sup _{Y} g \geq \alpha$, so that $\left(\mathrm{I}_{\alpha}\right)$ fails.

Proof of Lemma 2. To see that $\left(\mathrm{a}_{2}\right)$ implies $\left(\mathrm{d}_{2}\right)$ : If $\inf _{X} f=-\infty$, then $g(y) \geq \inf _{X} f$ is satisfied by each $y \in Y$. If $\inf _{X} f>-\infty$, then $\sim\left(\mathrm{I}_{a}\right)$ holds for $\alpha=\inf _{X} f$, and hence $\left(a_{2}\right)$ implies $g(y) \geq \alpha$ for some $y \in Y$. By the choice of $\alpha$, this gives $\left(d_{2}\right)$. To see that $\left(d_{2}\right)$ implies $\left(a_{2}\right)$ : Let $a \in$ $(-\infty,+\infty)$ be such that $\sim\left(I_{\alpha}\right)$ holds, that is, $\inf _{X} f \geq a$. Then by $\left(\mathrm{d}_{2}\right)$ there exists some $y \in Y$ such that $g(y) \geq \alpha$, so that (II $\alpha$ ) holds.

We envision applying the Proposition primarily to situations in which both $X$ and $Y$ are nonempty, with whatever "constraints" are present built in to $f$ and $g$ via the use of $+\infty$ and $-\infty$. It may be of interest to note, though, that with only a slight modification everything applies even allowing $X$ or $Y$ to be empty. One simply uses wherever necessary the conventions inf $\varnothing=+\infty$ and $\sup \varnothing=-\infty$, and in Lemma 2 one modifies statement $\left(\mathrm{d}_{2}\right)$ by adding the clause "or else $Y=\not \supset$ and $\inf _{X} f=-\infty$." Then $(\mathcal{P})$, with attainment in the dual problem interpreted as true vacuously, is still equivalent to $\left(\mathrm{d}_{1}\right)$ and $\left(\mathrm{d}_{2}\right)$, and all the proofs remain valid.

\section{REFERENCES}

1. M. L. Balinski and A. W. Tucker, Duality theory of linear programs: A constructive approach with applications, SIAM Rev. 11 (1969), 347-377. MR 41 \#3098.

2. O. L. Mangasarian, Nonlinear programming, McGraw-Ilill, New York, 1969. MR $40 \# 5263$. 
3. L. McLinden, Transposition theorems, old and new (in preparation).

4. R. T. Rockafellar, Convex analysis, Princeton Math. Ser., no. 28, Princeton Univ. Press, Princeton, N. J., 1970. MR 43 \#445.

5. - Conjugate duality and optimization, CBMS Regional Conf. Ser., no. 16, SIAM, Philadelphia, Pa., 1975.

6. J. Stoer and C. Witzgall, Convexity and optimization in finite dimensions. I, Die Grundlehren der math. Wissenschaften, Band 163, Springer-Verlag, Berlin and New York, 1970. MR 44 \#3707.

DEPARTMENT OF MATHEMATICS, UNIVERSITY OF ILLINOIS, URBANA, ILLINOIS 61801 